\title{
Discrepancy in results between dipstick urinalysis and urine sediment microscopy
}

\author{
ANCA BACÂREA ${ }^{1}$, GYULA LÁSZLÓ FEKETE ${ }^{2}$, \\ BIANCA LIANA GRIGORESCU ${ }^{1}$ and VLADIMIR CONSTANTIN BACÂREA ${ }^{3}$
}

\begin{abstract}
Departments of ${ }^{1}$ Pathophysiology and ${ }^{2}$ Dermatology, Dermatology Clinic, ${ }^{3}$ Department of Medical Research Methodology, 'George Emil Palade' University of Medicine, Pharmacy, Science and Technology, 540139 Targu Mures, Romania
\end{abstract}

Received January 29, 2021; Accepted March 1, 2021

DOI: $10.3892 /$ etm.2021.9971

\begin{abstract}
With the advancement of urine test automation and the large-scale application of quality management policies, the source of the most crucial errors has become the pre-analytical phase. This study is an attempt to compare the results obtained from the examination of urine strips with those obtained by microscopic examination of urinary sediment, highlighting discordant results. This observational study was conducted between February and August 2019 in a private medical laboratory in Mureş County, and 2,600 urine samples were analyzed. We calculated the sensitivity, specificity, positive predictive value, negative predictive value for leukocytes, nitrites and red blood cells, taking as reference the microscopic examination of urine summary screening. Urine samples were collected from patients who presented to the laboratory. The 2,600 urine samples were analyzed using strips with 10 parameters: glucose, protein, bilirubin, urobilinogen, $\mathrm{pH}$, specific density, red blood cells, nitrite, and leukocytes, and then using the microscope to examine the urinary sediment. We identified a small percentage $(1.92 \%)$ of inconsistencies from the 2,600 samples of urine, between urinalysis and the microscopic examination and we identified the causes. The most common discordant results were: false-negatives for nitrite $(72 \%)$, followed by false-positives results for red blood cells (22\%), false-negative results for leukocytes (16\%), false-negative results for red blood cells (4\%) and false-positives for leukocytes (4\%). The study confirmed that discrepancies appear despite the proper instruction of patients.
\end{abstract}

Correspondence to: Dr Gyula László Fekete, Department of Dermatology, Dermatology Clinic, 'George Emil Palade' University of Medicine, Pharmacy, Science and Technology, 38 Gheorghe Marinescu Street, 540139 Targu Mures, Romania

E-mail:dermafek@yahoo.com

Key words: dipstick urinalysis, microscopy, $\mathrm{pH}$, urine sediment, discordant results

\section{Introduction}

Urine is a biological product of great importance in clinical diagnosis, presenting important changes in various renal or non-renal diseases. With the advancement in urine test automation and the large-scale application of quality management policies, the source of the most crucial errors has become the pre-analytical phase (1). Obtaining accurate results is dependent on the clinician's understanding of the principles under which urine testing is carried out. Identification and monitoring of biochemical alterations or pathological elements in the urinary sediment may provide important clinical data on a patient's pathology (2).

Despite the existing guidelines, the importance of the appropriate method for urine collection is not recognized by the patient and often not followed. It has been shown that urine samples (first, middle stream morning urine and $24 \mathrm{~h}$ urine) are not properly collected in more than half of cases even when they are previously instructed, and especially in elderly patients with chronic kidney disease $(3,4)$. The urine specimen should be analyzed in less than two hours after collection, as the delay in the examination often yields erroneous results (5). Failure to comply to the standards for collecting, transporting, and storing urine samples results in pre-analytical errors that interfere with accurate and reliable results (6). In medical practice, there are often inconsistencies between the results of the urine screening summary and the urine sediment test results.

The aim of the present study was to compare the results obtained in urinalysis with urine strips with those obtained in the microscopic examination of the urinary sediment, to highlight the discrepancies and to try to explain them.

\section{Materials and methods}

This observational study was conducted between February and August 2019 in a private medical laboratory in Reghin, Mureş County, Romania, with the approval of the Ethics Committee of the Mures College of Physicians, and it follows the Helsinki Declaration principles.

Urine samples were collected from patients who presented to the laboratory; some of which were collected at the laboratory, and others were collected by the patients at home. Patients 
Table I. Causes of discordant results between between dipstick urinalysis and urine sediment microscopy.

\begin{tabular}{lrr}
\hline Causes of discordant results & No. & $\%$ \\
\hline Consumption of vitamin C & 13 & 26.0 \\
Infection with Staphylococcus saprophyticus (evidenced by urine culture) & 5 & 10.0 \\
Urinary density <1.005 & 5 & 10.0 \\
Urinary density >1.030 & 4 & 8.0 \\
Infection with Escherichia coli (evidenced by urine culture) & 4 & 8.0 \\
Antibiotic treatment (not declared or not known) & 4 & 8.0 \\
Incorrect harvest of urine-urine has been in the bladder for less than $2 \mathrm{~h}$ & 4 & 8.0 \\
Enterococcus spp. infection (evidenced by urine culture) & 3 & 6.0 \\
Leukocytes in the urine are lymphocytes, which do not contain leukocyte esterase & 2 & 4.0 \\
Treatment with cephalosporin & 2 & 4.0 \\
Urine with acid pH & 2 & 4.0 \\
Over glycosuria & 1 & 2.0 \\
Urine with alkaline pH & 1 & 2.0 \\
Total & 50 & 100.0 \\
\hline
\end{tabular}

Table II. Sensitivity and specificity for dipstick urinalysis parameters.

\begin{tabular}{lccccc}
\hline Parameter & Sensitivity (95\% CI) & Specificity (95\% CI) & PPV (95\% CI) & NPV (95\% CI) & P-value \\
\hline Leukocytes & $0.998(0.995-0.999)$ & $0.995(0.971-0.993)$ & $0.995(0.991-0.998)$ & $0.996(0.987-0.999)$ & $<0.0001$ \\
Nitrites & $0.998(0.996-0.999)$ & $0.940(0.918-0.957)$ & $0.980(0.972-0.986)$ & $0.996(0.987-0.000)$ & $<0.0001$ \\
RBCs & $0.996(0.992-0.998)$ & $0.994(0.984-0.998)$ & $0.998(0.995-0.999)$ & $0.989(0.977-0.996)$ & $<0.0001$ \\
\hline
\end{tabular}

PPV, positive predictive value; NPV, negative predictive value; RBCs, red blood cells.

were trained to appropriately collect their urine sample by the doctors employed at the laboratory. Those who collected urine at home received prior written urine collection instructions. The patients also received sterile containers from the laboratory. The first morning urine was collected from the middle stream, after appropriate hygienic measures.

We analyzed 2,600 urine samples using the Arkray Semi-Automated Analyzer and using Aution Sticks 4EA strips with 10 parameters including glucose, protein, bilirubin, urobilinogen, $\mathrm{pH}$, specific density, red blood cells, nitrite, and leukocytes, and then using the Nikon Eclipse E100 microscope to examine the urinary sediment (7). All urine specimens were examined using both strip and microscope within two hours. Tables with the limits and correspondence between the quantitative and qualitative value of the Aution Sticks strips were used for the chemical examination of the urine.

Criteria for the inclusion of urine samples were: i) first morning urine from the middle stream, after appropriate hygienic measures; ii) collection in sterile containers as recommended and provided by the laboratory staff. The criteria for the exclusion of urine samples were: i) the urine of women in the menstrual cycle due to possible erythrocyte contamination; ii) antibiotic use; iii) samples taken in inappropriate containers or non-compliant patients; iv) insufficient urine volume; v) visibly contaminated sample (e.g., feces); v) inappropriate urine storage; vi) failing to label the sample.
Statistical analysis was carried out with SPSS 20 (Statistical Package for Social Sciences; IBM Corp.). We used elements of descriptive statistics: frequencies, percentages, and confidence intervals (CIs). We used contingency tables for the calculation of sensitivity, specificity, positive predictive value (PPV), negative predictive value (NPV). The Chi-square test was used to calculate statistical significance. A P-value $<0.05$ was considered to indicate a statistically significant difference.

\section{Results}

Of the 2,600 subjects surveyed, only 50 (1.92\%) showed discrepancies, between biochemical examination with strips and microscopic examination of the urinary sediment.

The samples that showed discrepancies were from subjects of both sexes: 11 males $(22 \%)$ and 39 females (78\%). The average age of the patients was 47.50 years, with an age range of 8-78 years.

The most common diagnoses associated with discrepant samples were urinary tract infections (36\% of cases), followed by diabetes (18\%), hypertension (10\%) and anemia (8\%). These diagnoses were established by the family doctor or specialist.

The most common discordant results were: False-negatives for nitrite $(72 \%)$, followed by false-positive results for red blood cells (22\%), false-negative results for leukocytes (16\%), false-negative results for red blood cells (4\%) and false-positive 
results for leukocytes (4\%). The most important causes of the discrepancies are documented in Table I.

We calculated the sensitivity, specificity, PPV, and NPV for the parameters showing discordant results, taking as reference the microscopic examination of urine summary. The results are presented in Table II.

We obtained high values for sensitivity and specificity for all three parameters. In addition, PPV and NPV were high, indicating that dipstick urinalysis is a reliable test.

\section{Discussion}

The study confirmed that discrepancies do appear despite the proper instructions given to patients regarding urine collection procedure. Numerous factors can affect the urine test (diet, exercise, hydration, medication), and patient training is needed. Sometimes despite proper training, some patients are not compliant (e.g., not declaring or not knowing about antibiotic use). Regarding this issue, we discuss why two subjects included in the study, although they were instructed regarding correct sample collection, did not know that the drugs they were using were antibiotics (cephalosporin). We did not exclude these two patients as they initially met the inclusion criteria and we wanted to emphasize this possibility of this type of discordance.

Some patients, especially in during the cold and flu season, consume large amounts of vitamin $\mathrm{C}$ in the form of dietary supplements or in the form of foods and beverages containing vitamin $\mathrm{C}$ having a preservative and antioxidant effect. Examination of ascorbic acid interference has shown that the intensity of interference differs between dipsticks. Ascorbic acid interferes with glucose, hemoglobin, nitrite, and bilirubin at different concentrations causing false-negative results $(8,9)$.

The specificity of red blood cell (RBC) testing can be limited, providing false-positive results and this aspect should be taken into account in the diagnosis of hematuria. In alkaline urine $(\mathrm{pH}>7.0)$ or diluted urine (density $<1.010)$, RBCs can be lysed with hemoglobin release $(3,10)$. Strip testing of RBCs is influenced by ascorbic acid and gentisic acid (found in certain plants or drugs) (9). Hemoglobinuria, myoglobinuria, menstrual blood, concentrated urine, and strenuous exercise can cause a false-positive result on a dipstick test (4). A $\mathrm{pH}<5.1$ and increased urinary density may result in false-negative reactions on the strip (4). Strong oxidizing agents, soaps, detergents, sodium hypochlorite, hydrogen peroxide directly oxidize chromogen and produce a reaction in the absence of hemoglobin, giving a false-positive result. In addition, microbial peroxidase produced by certain bacteria such as Escherichia coli can catalyze the reaction in the absence of hemoglobin peroxidase with false-positive results. Thus, dipstick hematuria must be confirmed by microscopic examination to rule out false-positive and false-negative results (2).

Many of these discrepancies arise because patients presenting to the physician with symptoms of kidney disease, especially urinary tract infections, are treated with antibiotics before they perform a urine test. Leukocytes are particularly sensitive in hypotonic urine (density <1.010) and in alkaline urine $(\mathrm{pH}>7.0)$, being lysed (11). In the presence of bacteriuria, high temperature preservation and inappropriate centrifugation, leukocytes can also be lysed (4).
False-negative results may occur when the elevated leukocyte count in urine is due to lymphocytes; the test is negative on the strip but positive for microscopic examination. Also, the presence of substances that reduce the sensitivity of the reaction to leukocyte esterase (glucose in urine $>3 \mathrm{~g} / \mathrm{dl}$ and proteins $>500 \mathrm{mg} / \mathrm{dl}$, ketone bodies) or strong oxidizing agents, and antibiotics such as gentamicin, nitrofurantion or tetracycline can give false results.

The leukocyte esterase test provide false-positive reactions in case of the use of strong oxidizing agents or colored urine (bilirubinuria). A source of confusion can be the contamination of urine with vaginal mucus or secretion and also the use of certain drugs (nitrofurantion, clavulanic acid, meropenem and imipenem) $(4,12)$.

Detection of nitrites can be reduced, resulting in false-negative results due to antibiotic therapy that inhibits the conversion of nitrates to nitrites as well as the presence of ascorbic acid, $\mathrm{pH}<6.0$ and increased urinary density. Non-nitrate-reducing organisms also may cause false-negative results, and patients who consume a low-nitrate diet may have false-negative results (5).

Incorrect sample collection may be associated with contamination. According to Pernille et al the main contaminants in first-void urine samples were Enterococcus spp., which contributes to the majority of false-positives results (13). Inappropriate handling and preservation of the urine sample can cause bacterial proliferation and nitrite formation in vitro (3). A negative test for nitrites does not exclude urinary infection, and a positive result for nitrite should indicate bacteriological cultures (14).

Despite the discrepancies mentioned above, we obtained very good sensitivity and specificity for leukocytes, nitrites and RBCs for dipstick analysis when compared with the microscopic examination of urine sediment, making this test appropriate for urine analysis. According to Miler and Nikolac (15), there is no risk for the patients to exclude a microscopic exam, when dipstick testing is negative. This approach is also time saving (15). Bataille et al (16) showed that urinary dipstick qualifies as a better screening test for hematuria than urine microscopy analysis or flow cytometry, making dipstick urinalysis a good surrogate for urine microscopy.

One important utility of dipstick testing is to indicate a possible urinary infection. A positive sample (nitrates, leukocyte esterase) is then sent for urine culture (the gold standard). The question that arises is if the test is good enough for screening purposes. In early phases of uncomplicated urinary tract infections, when low bacterial counts are typical, both leukocyte esterase and nitrites may be undetectable.

Our research has some limitations. The samples were collected in a private laboratory and the numbers could be increased. In addition, the population who presented at the laboratory was variable in age and associated pathology. Similar studies have been commonly performed in hospitals in certain departments, in certain pathologies or age groups. One study assessed the accuracy of urine dipstick to predict urinary tract infections in an emergency department (17), and the sensitivity calculated for combined nitrite and leukocyte esterase test was $94 \%$ and the predictive value of nitrite and leukocyte esterase together for a negative urine culture was $95 \%$. Another study showed that positive nitrate is the best 
indicator of a positive culture with a specificity of $99.3 \%$ but had a negative likelihood ratio of 0.7 (18).

In a meta-analysis, Devillé et al (19) evaluated the accuracy of the urine dipstick test to rule out infections. The analysis of included studies showed high accuracy of nitrites in pregnant women and elderly individuals; PPV $\geq 80 \%$ in elderly and in family medicine. The highest sensitivity was reported in studies carried out in family medicine (90\%). Of course, the urine culture is the gold standard with which to diagnose urinary infection. Knowing these discordant results, the laboratories must ensure that they take the necessary steps to correct them.

The fact that we collected samples from the general population, regardless of age and associated pathology, brings value to our study, and our results may be useful to other laboratories. Despite proper training, some patients are not compliant with the urine collection procedure and this is an important pre-analytical source for false-negative or false-positive results.

In conclusion, we identified a small percentage (1.92\%) of inconsistencies between urinalysis and microscopic examination and our results confirm that dipstick urinalysis is an appropriate test.

\section{Acknowledgements}

We want to thank Dr Szekely Edit for reading and editing this article.

\section{Funding}

Not funding was received.

\section{Availability of data and materials}

The datasets used during the present study are available from the corresponding author upon reasonable request.

\section{Authors' contributions}

AB designed the study, performed the literature review and wrote the article. GLF participated in the creation of the database, wrote, read and corrected the article. BLG participated in the creation of the database, read and corrected the article. VCB designed the study, performed the statistical analysis of data, read and corrected the article. All authors read and approved the manuscript and agree to be accountable for all aspects of the research in ensuring that the accuracy or integrity of any part of the work are appropriately investigated and resolved.

\section{Ethics approval and consent to participate}

This study was approved by the Ethics Committee of the Mures College of Physicians (no. 4554) on July 25, 2019. The patients provided consent for the use of their data in the study.

\section{Competing interests}

The authors report no competing interests.

\section{References}

1. Clinical and Laboratory Standards Institute: Urinalysis and Collection, Transportation, and Preservation of Urine Specimens; Approved Guideline-Third Edition NCCLS document GP16-A3, http://www.clsi.org/. Accessed January 17, 2021.

2. Henderson SR and Harber M: Urine analysis. In: Practical Nephrology. Harber M (ed.), Springer-Verlag, London, pp19-29, 2014.

3. Brunzel NA: Elsevier Saunders, Quality Assurance and Safety, Urine Specimen Types, Collection, and Preservation, Chemical Examination of Urine, Microscopic Examination of Urine Sediment, Automation of Urine and Body Fluid Analysis. In: Fundamentals of Urine and Body Fluids Analysis, Elsevier, St. Louis, pp21-33, 37-44, 109-147, 157-210, 373-383, 2013.

4. Delanghe J and Speeckaert M: Preanalytical requirements of urinalysis. Biochem Med (Zagreb) 24: 89-104, 2014.

5. Simerville JA, Maxted W and Pahira JJ, Urinalysis: A comprehensive review. Am Fam Physician 71: 1153-1162, 2005.

6. Ercan M, Akbulut ED, Abuşoğlu S, Yilmaz FM, Oğuz EF, Topçuoğlu C, Öztekin V and Boğdaycioğlu N: Stability of urine specimens stored with and without preservatives at room temperature and on ice prior to Urinalysis. Clin Biochem 48: 919-922, 2015.

7. Fogazzi GB: Urinary sediment: Still an important diagnostic tool. Clin Chem Lab Med 53 (Suppl 2): S1451, 2015.

8. Unic A, Nikolac Gabaj N, Miler M, Culej J, Lisac A, Horvat A and Vrkic N: Ascorbic acid-a black hole of urine chemistry screening. J Clin Lab Anal 32: e22390, 2018.

9. Ko DH, Jeong TD, Kim S, Chung HJ, Lee W, Chun S and Min WK: Influence of vitamin c on urine dipstick test results. Ann Clin Lab Sci 45: 391-395, 2015.

10. Tomson $\mathrm{C}$ and Porter T: Asymptomatic microscopic or dipstick haematuria in adults: Which investigations for which patients? A review of the evidence. BJU Int 90: 185-198, 2002.

11. Mambatta AK, Jayarajan J, Rashme VL, Harini S, Menon S and Kuppusamy J: Reliability of dipstick assay in predicting urinary tract infection. J Family Med Prim Care 4: 265-268, 2015.

12. Patel HP: The abnormal urinalysis. Pediatr Clin North Am 53: 325-337, 2006.

13. Pernille H, Lars B, Marjukka M, Volkert S and Anne H: Sampling of urine for diagnosing urinary tract infection in general practice-first-void or mid-stream urine? Scand J Prim Health Care 37: 113-119, 2019.

14. Christenson RH, Tucker JA and Allen E: Results of dipstick tests, visual examination of urine sediment and microbiological culture of urine compared for simplifying urinalysis. Clin Chem 31: 448-450, 1985.

15. Miler M and Nikolac N: Patient safety is not compromised by excluding microscopic examination of negative urine dipstick. Ann Clin Biochem 55: 77-83, 2018.

16. Bataille A, Wetzstein M, Hertig A, Vimont S, Rondeau E and Galichon P: Evidence of dipstick superiority over urine microscopy analysis for detection of hematuria. BMC Res Notes 9: 435-437, 2016.

17. Rehmani R: Accuracy of urine dipstick to predict urinary tract infections in an emergency department. J Ayub Med Coll Abbottabad 16: 4-7, 2004.

18. Huysal K, Budak YU, Karaca AU, Aydos M, Kahvecioğlu S, Bulut M and Polat M: Diagnostic accuracy of urised automated urine microscopic sediment analyzer and dipstick parameters in predicting urine culture test results. Biochem Med (Zagreb) 23: 211-217, 2013.

19. Devillé WL, Yzermans JC, Van Duijn NP, Bezemer PD, van der Windt DA and Bouter LM: The urine dipstick test useful to rule out infections. A meta-analysis of the accuracy. BMC Urol 4: 4, 2004.

\section{Patient consent for publication}

Not applicable. 